\section{New insights into cataracts}

\section{By Lev Osherovich, Senior Writer}

A study by Case Western Reserve University researchers provides evidence for targeting the ephrin A2 receptor to treat age-related cortical cataracts, the most common cause of blindness. ${ }^{1}$ The question is whether to modulate the receptor's activity using mAbs or small molecules or to aim downstream at oxidative stress pathways controlled by the receptor.

The mouse and human genetic study was published in PLoS Genetics by a team co-led by Bingcheng Wang, professor of pharmacology, and Sudha Iyengar, assistant professor of epidemiology and biostatistics.

Cataracts result from the accumulation of misfolded proteins in the lens. Currently, the only treatment is lens replacement surgery. Although a rare hereditary form of cataract is caused by defects in a chaperone protein that keeps lenses free of misfolded protein debris, the causes of more common age-related cataracts are unknown, frustrating efforts to develop nonsurgical therapies.

"A therapy that targets an early stage of the disease in susceptible populations may push back the time before people would require surgery," said Iyengar.

The ephrin A2 receptor (EPHA2) is one of a large family of receptor tyrosine kinases best known as regulators of neuronal and vascular development. EPHA2 and similar proteins inhibit growth in response to contact by ligands presented by neighboring cells and have recently turned up in cancer studies as negative regulators of tumor growth and migration. ${ }^{2}$

The Case Western study suggests that both loss-of-function and gainof-function mutations in EPHA2 can cause cataracts.

Wang said EPHA2's connection with cataracts came to light while his team was characterizing the effect that deleting the gene had on cancer in mice.

"We generated Epha2 kinase knockout mice, and my technician noticed that the mice developed cataracts that progressed over time," similar to what happens in elderly humans, said Wang.

By 8 months, 74\% of Epha2 knockout mice had visible cataracts, whereas control mice with wild-type or heterozygous Epha2 had no cataracts. The lenses of knockout mice had higher levels of chaperone protein than those of wild-type controls, suggesting that the mutant lenses had problems with protein folding.
Wang and Iyengar's mouse data are consistent with a report published last year showing that mice lacking ephrin-A5 (EFNA5; AL-1), an inhibitory EPHA2 ligand, also develop cataracts. ${ }^{3}$

\section{Eye contact}

Meanwhile, Iyengar discovered the connection between EPHA2 mutations and cataracts in humans independently of Wang's mouse work. In 2004, Iyengar identified a region on chromosome 1 near the EPHA2 gene that was linked to higher risk of age-related cataracts. ${ }^{4}$

Based on Wang's mouse data, Iyengar suspected that EPHA2 was to blame, so the two researchers joined forces to characterize human EPHA2 variants linked with the disease.

In a meta-analysis of previous linkage studies, common SNPs within the EPHA2 gene were more highly associated with greater age-related cataract risk than nearby SNPs.

The breakthrough came when the team sequenced the EPHA2bearing chromosomal region in 34 members of a family with a history of cataracts.

Individuals bearing a missense mutation in the kinase domain of EPHA2 had significantly higher risk of cataracts than individuals without a mutation $\left(p=2 \times 10^{-8}\right)$. Because the affected individuals in this family were all heterozygotes, the EPHA2 mutation is likely to have a dominant, gain-of-function effect.

Indeed, when the team put the mutant EPHA2 into cultured human cells, they saw higher kinase activity and greater sensitivity to a growth-inhibiting EPHA2 ligand than that in cells transfected with wild-type EPHA2.

\section{Through a glass darkly}

On the face of it, the mouse and human data on EPHA2 point in different directions. In humans, excessive EPHA2 activity promotes cataracts. Thus, inhibiting EPHA2 might be useful for treating cataracts in genetically susceptible patients. On the other hand, because deletion of Epha2 in mice also leads to cataracts, excessive inhibition of EPHA2 may have an undesirable effect.

How best to modulate EPHA2 may depend on how common EPHA2linked SNPs affect the protein's activity, as well as whether EPHA2 activity is upregulated or downregulated in human cataract patients.

"You could either positively or negatively modulate EPHA2 to inhibit cataract development" depending on the specific molecular lesion in an individual, said Wang.

Wang's team has identified small molecule modulators of EPHA2 and plans to test them in mouse assays of cataract formation. He has filed patents for these compounds, which are available for licensing.

Besides targeting EPHA2 itself, Wang thinks that molecules downstream of the signaling protein also could make good targets.

For example, Wang and Iyengar's team found that Epha2 knockout mice had higher levels of oxidative stress than wild-type controls. Although the specific molecular effectors of this increased stress 


\section{TARGETS \& MECHANISMS}

response are not yet clear, Wang suspects cataracts could be treated with antioxidants.

\section{Keep an eye out}

The findings could be good news for the MedImmune Inc. subsidiary of AstraZeneca plc, which has an EPHA2 antibody in the clinic for cancer and has in-licensed IP from Purdue University covering the target in other indications.

Theresa LaVallee, director of translational science in oncology at MedImmune, cautioned that EPHA2 and its 13 close mammalian homologs "have a complicated biology," potentially making efforts to selectively target EPHA2 with small molecules more difficult.

"These proteins have roles primarily in the embryonic development of nervous and vascular tissues and, in normal adults, have roles in adhesion and vascular outgrowth," said LaVallee. The multitude of related targets raise the danger of off-target effects of putative EPHA2 modulators.

"Developing the right assays to assess specificity is tremendously important," she said.

MEDI-547, an EPHA2-binding human mAb conjugated to a cytotoxic compound, is in Phase I testing for solid tumors.

LaVallee said MEDI-547 doesn't affect EPHA2 activity per se, but rather uses the receptor as a dock to deliver the cytotoxic compound to selectively kill EPHA2-expressing tumor cells.

LaVallee said MedImmune might consider MEDI-547 for other indications besides cancer after evaluating the compound's performance in cancer trials.

Osherovich, L. SciBX 2(33); doi:10.1038/scibx.2009.1266

Published online Aug. 27, 2009

\section{REFERENCES}

1. Jun, G. et al. PLoS Genet.; published online July 31, 2009; doi:10.1371/journal.pgen.1000584

Contact: Bingcheng Wang, Case Western Reserve University School of Medicine, Cleveland, Ohio

e-mail: bxw14@case.edu

Contact: Sudha K. lyengar, same affiliation as above e-mail: ski@case.edu

2. Pasquale, E.B. Nat. Rev. Mol. Cell Biol. 6, 462-475 (2005)

3. Cooper, M.A. et al. Proc. Natl. Acad. Sci. USA 105, 16620-16625 (2008)

4. lyengar, S.K. et al. Proc. Natl. Acad. Sci. USA 102, 4485-14490 (2004)

COMPANIES AND INSTITUTIONS MENTIONED

AstraZeneca plc (LSE:AZN; NYSE:AZN), London, U.K. Case Western Reserve University, Cleveland, Ohio Medlmmune Inc., Gaithersburg, Md.

Purdue University, West Lafayette, Ind. 